\title{
Acute Thermotherapy Prevents Impairments in Cutaneous Microvascular Function Induced by a High Fat Meal
}

\author{
Jennifer C. Harvey, ${ }^{1}$ Bruno T. Roseguini, ${ }^{2}$ Benjamin M. Goerger, \\ Elizabeth A. Fallon, ${ }^{1}$ and Brett J. Wong ${ }^{1}$ \\ ${ }^{1}$ Department of Kinesiology \& Health, Georgia State University, Atlanta, GA 30302-3975, USA \\ ${ }^{2}$ Department of Health \& Kinesiology, Purdue University, West Lafayette, IN 47907, USA
}

Correspondence should be addressed to Brett J. Wong; bwong@gsu.edu

Received 12 January 2016; Revised 9 June 2016; Accepted 5 July 2016

Academic Editor: Andrea Flex

Copyright (C) 2016 Jennifer C. Harvey et al. This is an open access article distributed under the Creative Commons Attribution License, which permits unrestricted use, distribution, and reproduction in any medium, provided the original work is properly cited.

\begin{abstract}
We tested the hypothesis that a high fat meal (HFM) would impair cutaneous vasodilation, while thermotherapy (TT) would reverse the detrimental effects. Eight participants were instrumented with skin heaters and laser-Doppler (LD) probes and tested in three trials: control, HFM, and HFM + TT. Participants wore a water-perfused suit perfused with $33^{\circ} \mathrm{C}$ (control and $\mathrm{HFM}$ ) or $50^{\circ} \mathrm{C}(\mathrm{HFM}$ + TT) water. Participants consumed $1 \mathrm{~g}$ fat $/ \mathrm{kg}$ body weight. Blood samples were taken at baseline and two hours post-HFM. Blood pressure was measured every 5-10 minutes. Microvascular function was assessed via skin local heating from $33^{\circ} \mathrm{C}$ to $39^{\circ} \mathrm{C}$ two hours after HFM. Cutaneous vascular conductance (CVC) was calculated and normalized to maximal vasodilation $(\% \mathrm{CVC}$ max $)$. $\mathrm{HFM} \mathrm{had}$ no effect on initial peak $\left(48 \pm 4 \% \mathrm{CVC}_{\max }\right)$ compared to control $\left(49 \pm 4 \% \mathrm{CVC}_{\max }\right)$ but attenuated the plateau $(51 \pm 4 \% \mathrm{CVC}$ max $)$ compared to control $\left(63 \pm 4 \% \mathrm{CVC}_{\max }, P<0.001\right)$. Initial peak was augmented in $\mathrm{HFM}+\mathrm{TT}\left(66 \pm 4 \% \mathrm{CVC}_{\max }\right)$ compared to control and HFM $(P<0.05)$, while plateau $\left(73 \pm 3 \% \mathrm{CVC}_{\max }\right)$ was augmented only compared to the HFM trial $(P<0.001)$. These data suggest that HFM negatively affects cutaneous vasodilation but can be minimized by TT.
\end{abstract}

\section{Introduction}

Microvascular dysfunction, broadly defined as a state of enhanced vasoconstriction and reduced vasodilation, has been shown to play a key role in the progression of cardiometabolic-related diseases and thus it acts as an important contributor to chronic diseases such as diabetes, hypertension, and dyslipidemia [1-7]. Hypertriglyceridemia has been established as a risk factor of cardiovascular disease and it has been proposed that microvascular dysfunction precedes macrovascular dysfunction, thus making it important to understand how high fat meals/diets may affect human microvascular function [8-13].

The consumption of a high fat diet can transform acute postprandial microvascular endothelial dysfunction into a chronic vascular disease. High fat meal induced inflammation causes triglyceride-rich lipoproteins to accumulate in the blood. Chronic oxidative stress and the resulting chronic inflammation leads to a prolonged state of elevated circulating inflammatory markers furthering the risk for atherosclerosis and cardiovascular disease [14-17]. Nitric oxide (NO) is a vital molecule for cardiovascular health and vascular function as it regulates platelet aggregation and vascular tone [18-21]. Oxidative stress following a high fat meal may inhibit endothelial NO synthase (eNOS) phosphorylation, an important precursor for $\mathrm{NO}$ production in the peripheral vasculature $[18,22]$. In addition, antioxidant therapy has been shown to prevent the impairment in endothelial function, which further supports a role for oxidative stress [23-25]. Hypertriglyceridemia and the associated oxidative stress lead to the development of prediabetic peripheral neuropathy, particularly in the sensory afferent nerves [26]. High fat meals can thus negatively affect not only NO-dependent vasodilation but also sensory nerve function. Identifying physiologic systems and nonpharmacological interventions to stunt or prevent these effects are deemed important. 
The microvasculature is responsible for the bulk of glucose uptake and regulation of vascular resistance and is often the site of dysfunction that leads to diabetic neuropathy, all of which further underscores the importance of understanding how high fat meals affect microvascular function. A high fat meal can have an acute adverse effect, such as a reduction in whole limb blood flow as well as impaired pulmonary function; however, to our knowledge, there have been no studies directly investigating the effect of a high fat meal on human microvascular sensory nerve and endothelial function [27-30]. The cutaneous vasodilation in response to local skin heating (cutaneous thermal hyperemia) is a reproducible biphasic response: an initial, rapid vasodilation mediated primarily by sensory nerves that is followed by a prolonged plateau mediated largely $(\sim 80 \%)$ by NO derived from eNOS [31-41]. The cutaneous thermal hyperemic response can therefore be used to noninvasively assess microvascular sensory nerve function and endothelial NO-dependent vasodilation simultaneously [31, 32, 34-41].

Thermotherapy (TT) may have the potential to reduce the negative effects of a high fat meal on microvascular endothelial function and may ultimately provide a way to curb the development of atherosclerosis in a nonpharmaceutical manner by upregulating eNOS and inducing vasodilation of systemic arteries [42-45]. Elevated cardiac output concomitantly increases peripheral blood flow and, presumably, the amount of shear stress necessary to evoke NO production within the vessel [46]. Thus, sauna therapy appears to have remedial effects on microvascular endothelial function and in turn may act as a therapeutic modality for halting atherosclerotic processes [46].

The purpose of this study was to investigate the effect of a high fat meal on both microvascular sensory nerve function and microvascular endothelial function. A secondary purpose was to determine whether exposure to TT prior to a high fat meal could mitigate the detrimental effects of the high fat meal on microvascular function. Specifically, we tested two hypotheses: (1) a high fat meal will blunt both cutaneous microvascular sensory nerve- and endothelialmediated vasodilation and (2) TT application will reverse the detrimental effects of a high fat meal.

\section{Methods}

2.1. Ethical Approval. The Institutional Review Board at Georgia State University approved all protocols used in this study (number H14621). Written informed consent was reviewed and signed by all participants prior to participation in this study. All protocols were performed in accordance with the Declaration of Helsinki.

2.2. Participant Recruitment and Screening. Eight participants ( 4 males and 4 females; aged 18-32 years; BMI $22.8 \pm$ $0.3)$ were recruited and screened for health history prior to study participation. Vascular and oxidative stress responses to a high fat meal are affected by race/ethnicity. For example, studies have shown a greater detriment in vascular function in Asians compared to Caucasians following an acute high fat meal [23-25, 47-49]. Participants in the present study were Caucasian $(n=6)$ and African American $(n=2)$. None of the participants had any cardiovascular or metabolic disease, skin allergy/disease, and history of adverse reaction to heat stress, were smokers, or took any medication for corresponding conditions. For female participants, phase of menstrual cycle or oral contraceptive use was noted but not controlled for in these studies. Participants reported to the laboratory for a completion of three protocols: control, high fat meal (HFM), and high fat meal plus TT application (HFM-TT). The control protocol (no HFM and no TT) was performed first for each participant. The order of the two HFM protocols was randomized across participants. At least, one day, and no more than seven days, was allowed between the control protocol and the first HFM protocol. At least, seven days, and no more than 10 days, were allowed between the two HFM protocols. All experiments took place in a thermoneutral laboratory $\left(20^{\circ} \mathrm{C}-22^{\circ} \mathrm{C}\right.$ and $52-56 \%$ relative humidity).

2.3. Participant Instrumentation. For all protocols, a baseline fasting blood sample (at least eight hours fasted, water ad libitum) via finger stick was analyzed for triglycerides and glucose (Cholestech LDX, Alere; San Diego, CA, USA). Each participant wore a water-perfused suit to either clamp or raise core temperature (see details below). The water-perfused suit covered the entire body except head, hands, feet, and the experimental forearm. A core temperature pill (CorTemp, HQ Inc.; Palmetto, FL, USA) ingested two hours prior to laboratory testing was used to monitor core temperature, which was recorded every 3-5 minutes for the duration of the protocol. Each participant was equipped with a 3lead electrocardiogram and a blood pressure cuff was placed on the right arm. Blood pressure was measured via automated brachial oscillation every 5-10 minutes (Connex 6300, Welch-Allyn; Skaneateles Falls, NY, USA) for the duration of the protocol. An index of skin blood flow was measured at two sites on the dorsal aspect of the left forearm via laser-Doppler flowmetry and each laser-Doppler probe was housed within a local skin-heating unit that was used to control local skin temperature (moorVMS HEAT and LDF2, Moor Instruments; Devon, UK). Each local heater and laserDoppler probe was affixed to the skin with double-sided adhesive discs (Electrode Washers E432, In Vivo Metric; Healdsburg, CA, USA). Participants were seated in a recliner in the semirecumbent position with both arms at heart level for all protocols.

2.4. Local Heating Protocol. Both skin heaters were increased from $33^{\circ} \mathrm{C}$ to $39^{\circ} \mathrm{C}$ at a rate of $0.1^{\circ} \mathrm{C} /$ second to evoke submaximal vasodilation following a $10 \mathrm{~min}$ baseline [34]. This rate and magnitude of local heating have been shown to induce vasodilation that is $\sim 80 \% \mathrm{NO}$-dependent [36-39]. Once a plateau in skin blood flow was achieved (approximately 30 minutes of heating), the skin heaters were increased to $43^{\circ} \mathrm{C}$ to elicit maximal vasodilation $[31,32,34,40,41$, 50]. This heating protocol allows for assessment of both 


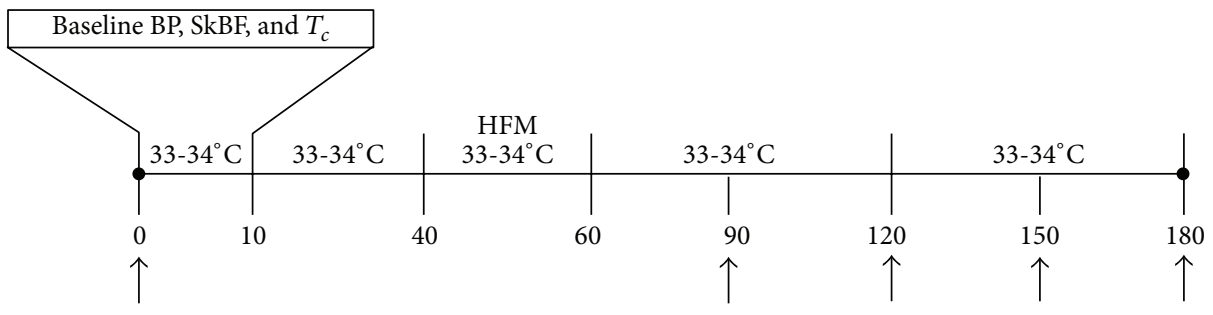

(a)

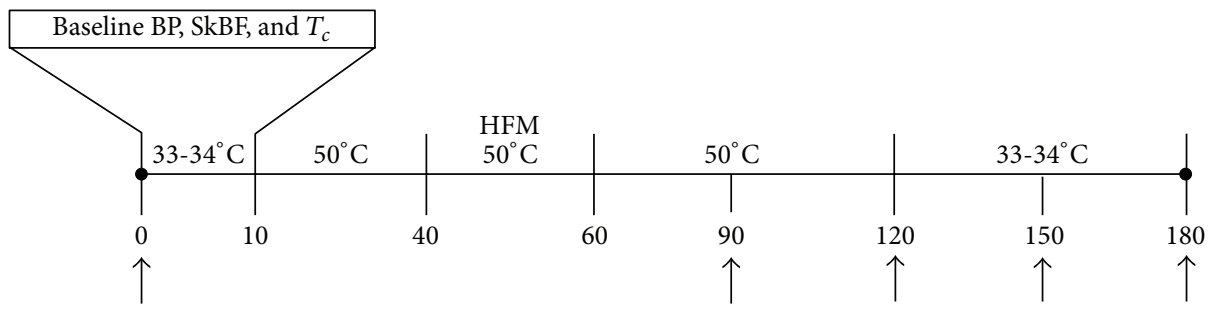

(b)

FIGURE 1: Schematic illustration of the experimental protocol. (a) HFM (thermoneutral) protocol and (b) HFM-TT protocol. Arrows indicate time points where finger stick blood samples were taken. Temperatures of $33-34^{\circ} \mathrm{C}$ (thermoneutral) and $50^{\circ} \mathrm{C}$ (thermotherapy) indicate the temperature of the water pumped through water-perfused suit. BP: blood pressure; SkBF: skin blood flow; $T_{c}$ : core temperature; HFM: high fat meal.

sensory nerve function and NO-dependent microvascular endothelial vasodilation $[11,34,39,51]$.

2.5. High Fat Meal (HFM). The high fat meal used in this study (ice cream) was based on two previous studies demonstrating that $1 \mathrm{~g}$ fat per kilogram body mass delivered via ingestion of ice cream attenuates pulmonary function and increases exhaled NO, a marker of pulmonary inflammation $[27,30]$. Fat consumption for each participant was calculated as one gram of fat per kilogram of body weight (serving size: $88 \mathrm{~g}$, total fat: $9 \mathrm{~g}$; $[27,30])$. Servings were measured via electronic balance scale (Ohaus, Scout Pro; Parsippany, NJ, USA) to the nearest one-hundredth of a gram. Participants consumed on average $672.43 \pm 53.70 \mathrm{~g}$ of ice cream. Each serving of ice cream provided $6 \mathrm{~g}$ saturated fat, $35 \mathrm{mg}$ cholesterol, $20 \mathrm{~g}$ carbohydrate, and $4 \mathrm{~g}$ protein. Participants were allotted 20 minutes to consume the ice cream.

2.6. Experimental Protocol 1: Control (No HFM, Thermoneutral). Following a baseline blood sample, participants underwent an initial local heating protocol as described above in order to assess their control sensory nerve function and NO-dependent vasodilation in the absence of a HFM. The water-perfused suit was perfused with thermoneutral water (33-34 ${ }^{\circ}$ C; Sahara S21 Stainless-Steel Heated Bath Circulator, ThermoFisher Scientific; Waltham, MA USA) to clamp core temperature.

2.7. Experimental Protocol 2: High Fat Meal (Thermoneutral). The experimental protocol is shown in Figure 1(a). Participants reported to the laboratory and underwent a fasting blood sample and rested for $10 \mathrm{~min}$ in the semirecumbent position, during which baseline data was acquired. Next, the water-perfused suit was connected to the water pump and perfused with water at $33^{\circ} \mathrm{C}$ for 30 minutes to clamp core temperature. After 30 minutes, participants consumed the high fat meal within the 20 minutes allotted. Upon completion of the high fat meal, participants underwent an additional two hours of exposure to thermoneutral water. At the end of the control intervention, a second finger stick blood sample was taken. Previous studies have shown endothelial dysfunction to occur two hours after a high fat meal [1-3].

2.8. Experimental Protocol 3: High Fat Meal + Thermotherapy. The experimental protocol is shown in Figure 1(b). This protocol was the same as protocol two (above) with the exception that participants underwent TT application instead of a control intervention. Thermotherapy was applied by pumping $50^{\circ} \mathrm{C}$ water through the water-perfused suit for 110 minutes: 30 minutes prior to the high fat meal, during HFM consumption $(20 \mathrm{~min})$, and 60 minutes after high fat meal. The goal of this protocol was to increase core temperature $\sim 0.2-0.3^{\circ} \mathrm{C}$ above baseline and also increase skin blood flow while avoiding pronounced vasodilation that could affect the subsequent local heating response (i.e., a ceiling effect due to elevated skin blood flow). Extensive pilot work was performed to determine that this duration of heating was sufficient to increase skin blood flow above baseline and increase core temperature $\sim 0.2-0.3^{\circ} \mathrm{C}$ above baseline core temperature. At the end of the $90 \mathrm{~min}$ of TT application, the temperature of the water was reduced to $33-34^{\circ} \mathrm{C}$ to allow the participant's core temperature and skin blood flow return to thermoneutral levels. Blood samples were taken thirty minutes after completing the high fat meal and every 30 minutes thereafter until the end of the protocol. 
2.9. Data Analysis. All data were sampled at $100 \mathrm{~Hz}$ (PowerLab 16/35, ADInstruments; Colorado Springs, CO, USA) and stored to the hard drive of a laboratory computer (iMac, Apple; Cupertino, California, USA). Cutaneous vascular conductance (CVC) was calculated as laser-Doppler flux divided by mean arterial pressure and was normalized as a percentage of maximal vasodilation $\left(\% \mathrm{CVC}_{\max }\right)$. There were no observable or statistical differences in $\% \mathrm{CVC}_{\max }$ between the two laser-Doppler sites within subjects for a given trial so values from each site were averaged.

Baseline values across trials and at various time points within a trial were compared to ensure that changes in $\% \mathrm{CVC}_{\max }$ were not due to baseline shifts in response to either the high fat meal or the mild heat stress. For the control trial, the pre-local heating baseline was averaged over the three-minute period immediately preceding the local heating protocol. For both the HFM and HFM-TT trial, three different time points were analyzed: (1) pre-high fat meal baseline was taken as the three-minute period immediately preceding initiation of either the thermoneutral period for the HFM trial or mild heat stress for the HFM-TT trial; (2) posthigh fat meal baseline was taken as the three-minute period immediately preceding the end of the thermoneutral period or the mild heat stress; and (3) pre-local heating baseline was taken as the three-minute period immediately preceding the beginning of the local heating protocol.

For the local heating responses, $\% \mathrm{CVC}_{\max }$ data were analyzed as follows. The initial peak is a rapid and transient response; data were thus averaged over a 30-60-second period corresponding to the highest values prior to a decrease in $\% \mathrm{CVC}_{\max }$ (onset of the nadir) of the local heating response $[33-35,40,41]$. Data for the plateau were averaged over a stable 3-5-minute period when the local heaters were held at $39^{\circ} \mathrm{C}[31,33,34,40,41]$. For maximal $\mathrm{CVC}$, data were averaged over a stable three-minute period when the local heaters were at $43^{\circ} \mathrm{C}$.

Blood samples were analyzed for differences between baseline during the control, HFM, and HFM-TT trials. The baseline values were compared to the values two hours after the high fat meal in the HFM and HFM-TT trials.

All data were analyzed via SPSS 22 (IBM Corporation; Armonk, NY, USA) and presented as mean \pm SEM. Statistical significance was initially set at $\alpha=0.05$, with Bonferroni corrections when appropriate. A Shapiro-Wilk test was performed to determine if the data were normally distributed. All data were normally distributed; however, only $P$ values for blood triglycerides are reported because of the large variability of the blood triglyceride data. One-way ANOVAs were used to examine differences in (a) baseline levels of TRG and GLU across experimental conditions, (b) baseline systolic, diastolic, and mean arterial blood pressure across experimental conditions, (c) changes in HR across conditions, and (d) $\% \mathrm{CVC}_{\max }$ across experimental conditions. Repeated measures ANOVAs were used to compare change in (a) TRG and GLU before and after HFM, (b) $\% \mathrm{CVC}_{\max }$ before and after HFM-TT, and (c) changes in core temperature during the HFM-TT trial.

A linear regression was conducted to examine the unique influence of blood triglycerides and blood glucose
TABLE 1: Blood triglyceride and blood glucose data. Both blood triglycerides and blood glucose increased above baseline in both high fat meal trials. HFM: high fat meal; HFM-TT: high fat meal plus thermotherapy. ${ }^{*} P<0.0125$ versus baseline within the same trial. Values are means \pm SEM.

\begin{tabular}{lcccc}
\hline & \multicolumn{2}{c}{ Blood triglycerides (mg/dL) } & \multicolumn{2}{c}{ Blood glucose (mg/dL) } \\
& Baseline & $\begin{array}{c}\text { After high fat } \\
\text { meal }\end{array}$ & Baseline & $\begin{array}{c}\text { After high fat } \\
\text { meal }\end{array}$ \\
\hline Control & $84 \pm 9$ & & $83 \pm 5$ & \\
HFM & $88 \pm 6$ & $178 \pm 16^{*}$ & $84 \pm 3$ & $105 \pm 3^{*}$ \\
HFM- & $91 \pm 9$ & $173 \pm 14^{*}$ & $82 \pm 3$ & $102 \pm 4^{*}$ \\
TT & & & \\
\hline
\end{tabular}

on $\% \mathrm{CVC}_{\max }$ for each trial (control, HFM, or HFM-TT) and for each phase of the local heating response (initial peak and plateau). Pearson correlations denote unadjusted relationships between blood triglycerides and $\% \mathrm{CVC}_{\max }$ and between blood glucose and $\% \mathrm{CVC}_{\max }$. Standardized beta coefficients denote associations between (a) blood triglycerides and $\% \mathrm{CVC}_{\max }$ after adjusting for blood glucose and (b) blood glucose and $\% \mathrm{CVC}_{\max }$ after adjusting for blood triglycerides. Thus, the advantage of beta coefficients is their ability to show the unique contribution of each blood variable (triglycerides or glucose) to the variance of $\% \mathrm{CVC}_{\max }$.

\section{Results}

3.1. Blood Variables. Blood triglyceride and glucose data for all protocols are shown in Table 1. Despite the high variability, all blood triglyceride data were normally distributed as determined from the Shapiro-Wilk test. There were no differences in baseline blood triglycerides between trials (control: $84 \pm$ $9 \mathrm{mg} / \mathrm{dL}$; HFM: $88 \pm 6 \mathrm{mg} / \mathrm{dL}$; HFM-TT: $91 \pm 9 \mathrm{mg} / \mathrm{dL} ; P=$ 1.000 for all conditions). There was a significant increase in blood triglycerides two hours after the high fat meal compared to baseline for both the HFM trial $(178 \pm 16 \mathrm{mg} / \mathrm{dL}$, $P=0.003)$ and the HFM-TT trial $(173 \pm 14 \mathrm{mg} / \mathrm{dL}, P=$ $0.002)$. There were no differences between the two high fat meal trials $(P=1.000)$. Baseline blood glucose did not differ between trials (control: $83 \pm 5 \mathrm{mg} / \mathrm{dL}$; HFM: $84 \pm 3 \mathrm{mg} / \mathrm{dL}$; HFM-TT: $82 \pm 3 \mathrm{mg} / \mathrm{dL} ; P=1.000$ for all conditions). Two hours after the high fat meal, there was a significant increase in blood glucose for both the HFM $(105 \pm 3 \mathrm{mg} / \mathrm{dL} ; P=0.019)$ and the HFM-TT $(102 \pm 4 \mathrm{mg} / \mathrm{dL} ; P=0.010)$ trials; however, there was no difference in blood glucose between the two high fat meal trials $(P=1.000)$.

3.2. Blood Pressure and Heart Rate. Blood pressure and heart rate data for all protocols are summarized in Table 2. Although systolic blood pressure during the HFM tended to be higher compared to the control trial $(P=0.078)$, this did not reach statistical significance. There were no statistical differences in systolic blood pressure between any of the other conditions (control versus HFM-TT: $P=0.975$; HFM versus HFM-TT: $P=0.511$ ). There were no statistical differences in either diastolic blood pressure between trials (control versus HFM: $P=0.912$; control versus HFM-TT: 


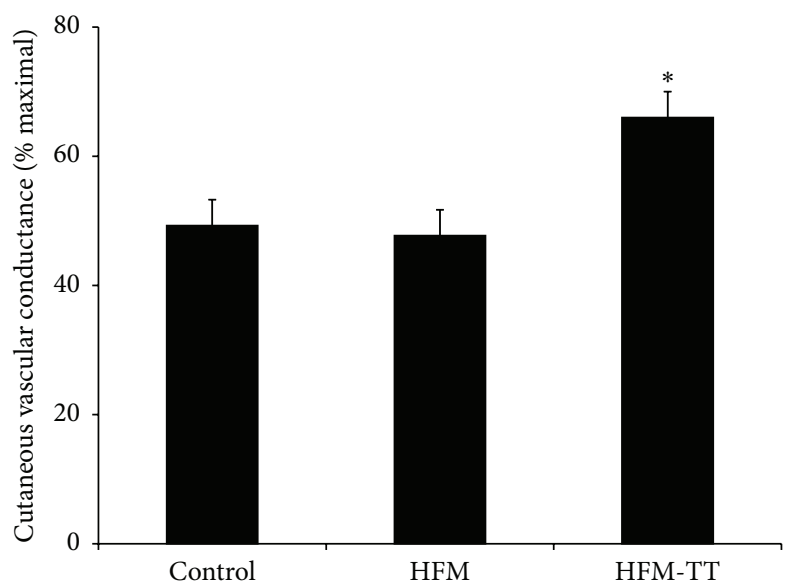

FIgURE 2: Initial peak $\% \mathrm{CVC}_{\max }$. Initial peak $\% \mathrm{CVC}_{\max }$ for the control, high fat meal (HFM), and high fat meal plus TT (HFM-TT) trials. There was no effect of the HFM on the initial peak compared to the control trial. Initial peak was augmented in the HFM-TT trial compared to the control and HFM trials. ${ }^{*} P<0.05$ versus control and HFM trials. Data are group mean \pm SEM.

TABLE 2: Blood pressure and heart rate data. There were no differences in any of the blood pressure values between the three trials. HFM: high fat meal trial; HFM-TT: high fat meal plus thermotherapy trial. Values are means \pm SEM. ${ }^{*} P<0.05$ versus control and HFM conditions.

\begin{tabular}{lcccc}
\hline & $\begin{array}{c}\text { Systolic } \\
\text { blood } \\
\text { pressure } \\
(\mathrm{mmHg})\end{array}$ & $\begin{array}{c}\text { Diastolic } \\
\text { blood } \\
\text { pressure } \\
(\mathrm{mmHg})\end{array}$ & $\begin{array}{c}\text { Mean arterial } \\
\text { blood } \\
\text { pressure } \\
(\mathrm{mmHg})\end{array}$ & $\begin{array}{c}\text { Heart rate } \\
\text { (beats/minute) }\end{array}$ \\
\hline Control & $116 \pm 5$ & $76 \pm 5$ & $89 \pm 5$ & $61 \pm 2$ \\
HFM & $120 \pm 5$ & $77 \pm 5$ & $91 \pm 5$ & $68 \pm 3$ \\
HFM-TT & $118 \pm 6$ & $75 \pm 4$ & $90 \pm 4$ & $88 \pm 3^{*}$ \\
\hline
\end{tabular}

$P=1.000$; HFM versus HFM-TT: $P=1.000)$ or in MAP between trials (control versus HFM: $P=0.300$; control versus HFM-TT: $P=1.000$; HFM versus HFM-TT: $P=0.868)$. Heart rate increased during the HFM-TT condition $(88 \pm 3$ beats/minute) compared to both control ( $61 \pm 2$ beats/minute; $P<0.05)$ and HFM $(68 \pm 3$ beats/minute; $P<0.05)$ conditions. Heart rate was not statistically different between control and HFM conditions $(P=0.817)$.

3.3. Core Temperature. Core temperature averaged $37.31 \pm$ $0.11^{\circ} \mathrm{C}$ throughout the control trial and $37.27 \pm 0.10^{\circ} \mathrm{C}$ for the duration of the HFM trial. For the HFM-TT trial, core temperature increased from a baseline of $37.36 \pm 0.13^{\circ} \mathrm{C}$ to $37.68 \pm 0.18^{\circ} \mathrm{C}$ during exposure to TT. There was no statistical difference in baseline core temperature between the three trials. The heat stress during the HFM-TT trial was sufficient to significantly raise core temperature to the goal of $\sim 0.2-$ $0.3^{\circ} \mathrm{C}$ above baseline $(P=0.042)$.

3.4. Baseline and Maximal Cutaneous Vascular Conductance. Baseline $\% \mathrm{CVC}_{\max }$ data for all three trials is shown in Table 3. There were no statistical differences between the prelocal heating baselines for any of the three trials, suggesting that changes in baseline cannot explain the observed differences
TABle 3: Baseline $\% \mathrm{CVC}_{\max }$ data. Baseline values for the three experimental trials. Pre-HFM was defined as the three-minute period just prior to either the thermoneutral or TT; post-HFM was defined as the three-minute period immediately preceding the end of either the thermoneutral or TT; pre-local heating was defined as the three-minute period just prior to beginning the local heating protocol. HFM: high fat meal; HFM-TT: high fat meal plus mild heat stress trial. ${ }^{*} P<0.05$ versus same time point compared to HFM; ${ }^{\#} P<0.05$ versus pre-HFM and pre-local heating within the same trial. Values are means \pm SEM.

\begin{tabular}{lccc}
\hline & Control trial & HFM trial & HFM-TT trial \\
\hline Pre-HFM & & $14 \pm 2$ & $18 \pm 3$ \\
Post-HFM & & $13 \pm 2$ & $32 \pm 3^{* \#}$ \\
Pre-local heating & $17 \pm 3$ & $17 \pm 3$ & $21 \pm 5$ \\
\hline
\end{tabular}

in $\% \mathrm{CVC}_{\max }$ of the initial peak or plateau during the HFM and HFM-TT trials ( $P=1.000$ for all conditions). The posthigh fat meal baseline during the HFM-TT trial (i.e., at the end of TT application) was significantly greater than the prehigh fat meal baseline $(P=0.022)$, indicating that TT was sufficient to approximately double skin blood flow.

Absolute maximal CVC values averaged $2.68 \pm 0.38$ for the control trial, $2.47 \pm 0.28$ for the HFM trial, and $2.63 \pm 0.27$ for the HFM-TT trial. There were no statistical differences in maximal CVC between trials ( $P>0.951$ for all conditions).

3.5. Cutaneous Vascular Conductance: Initial Peak Responses. The group data for the initial peak responses are shown in Figure 2. Initial peak during the control trial averaged $49 \pm$ $4 \% \mathrm{CVC}_{\max }$. The initial peak during the HFM trial $(48 \pm$ $\left.4 \% \mathrm{CVC}_{\max }\right)$ was not different compared to the control trial $(P=1.000)$. The initial peak during the HFM-TT trial $(66 \pm$ $\left.4 \% \mathrm{CVC}_{\max }\right)$ was augmented compared to both the control $(P=0.002)$ and the HFM trials $(P=0.011)$.

3.6. Cutaneous Vascular Conductance: Plateau Responses. The group data for the plateau phase of the local heating 


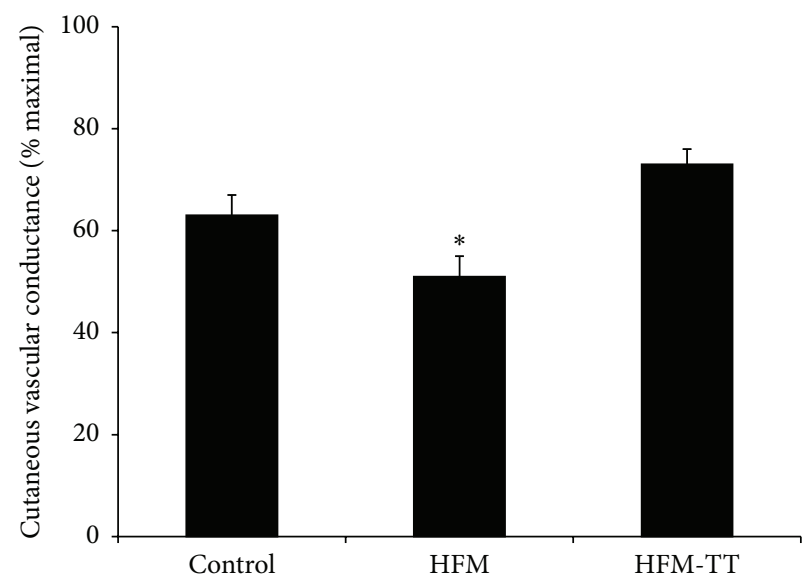

Figure 3: Plateau $\% \mathrm{CVC}_{\max }$. Plateau $\% \mathrm{CVC}_{\max }$ for the control, high fat meal (HFM), and high fat meal plus TT (HFM-TT) trials. Plateau in the HFM trial was attenuated compared to both the control and HFM-TT trials. There was no difference between the control and HFM-TT trials. ${ }^{*} P<0.05$ versus control and HFM-TT trials. Data are group mean \pm SEM.

TABLE 4: Linear regression for the correlation between $\% \mathrm{CVC}_{\max }$ and blood triglycerides and blood glucose for both the initial peak and plateau phase of cutaneous thermal hyperemia. HFM: high fat meal; HFM-TT: high fat meal + thermotherapy; TRG: blood triglycerides; GLU: blood glucose. * indicates statistically significant Pearson correlation and standardized beta coefficient.

\begin{tabular}{lcccccc}
\hline & \multicolumn{2}{c}{ Control } & \multicolumn{2}{c}{ HFM } & \multicolumn{2}{c}{ HFM-TT } \\
& TRG & GLU & TRG & GLU & TRG & GLU \\
\hline $\begin{array}{l}\text { Initial peak } \\
\begin{array}{l}\text { Pearson } \\
\text { correlation }\end{array}\end{array}$ & 0.424 & 0.252 & 0.267 & -0.145 & -0.268 & -0.433 \\
$P$ value & 0.148 & 0.273 & 0.262 & 0.366 & 0.260 & 0.142 \\
\hline $\begin{array}{l}\text { Standardized } \beta \\
\text { coefficient }\end{array}$ & 0.434 & 0.269 & 0.305 & -0.203 & -0.424 & -0.552 \\
$P$ value & 0.313 & 0.518 & 0.509 & 0.657 & 0.309 & 0.201 \\
\hline $\begin{array}{l}\text { Plateau } \\
\begin{array}{l}\text { Pearson } \\
\text { correlation }\end{array}\end{array}$ & 0.542 & 0.162 & -0.827 & 0.108 & -0.739 & 0.540 \\
$P$ value & 0.082 & 0.351 & $0.006^{*}$ & 0.399 & $0.018^{*}$ & 0.083 \\
\hline $\begin{array}{l}\text { Standardized } \beta \\
\text { coefficient }\end{array}$ & 0.550 & 0.183 & -0.879 & 0.275 & -0.637 & 0.361 \\
$P$ value & 0.195 & 0.639 & $0.01^{*}$ & 0.275 & 0.064 & 0.238 \\
\hline
\end{tabular}

response are shown in Figure 3. The $\% \mathrm{CVC}_{\max }$ values were significantly reduced in the HFM trial $\left(51 \pm 5 \% \mathrm{CVC}_{\max }\right)$ compared to the control $\left(63 \pm 5 \% \mathrm{CVC}_{\max } ; P<0.001\right)$ and the HFM-TT $\left(73 \pm 3 \% \mathrm{CVC}_{\max } ; P<0.001\right)$ trial. There was no statistical difference between the control and HRM-TT trials $(P=0.156)$.

3.7. Linear Regression Analysis of $\% C V C_{\max }$ with Blood Triglyceride and Blood Glucose. Linear regression results for the initial peak are shown in Table 4. Pearson correlations and standardized beta coefficients assessing the association between (a) the initial peak $\% \mathrm{CVC}_{\max }$ and blood triglycerides and (b) the initial peak and blood glucose for any of the experimental trials (control, HFM, or HFM-TT) were not statistically significant.

Linear regression results for the plateau are shown in Table 4. In the control trial, there were no statistically significant Pearson correlations or standardized beta coefficients. In the HFM trial, there was a statistically significant unadjusted negative association between plateau $\% \mathrm{CVC}_{\max }$ and blood triglycerides (Pearson correlation is $-0.827 ; P=0.006$ ). This association remained statistically significant after adjusting for blood glucose (standardized $\beta$ is $-0.879 ; P=0.011$ ). There was no statistically significant association between $\% \mathrm{CVC}_{\max }$ and blood glucose in the HFM trial. In the HFM-TT trial, there was a statistically significant unadjusted negative association between plateau $\% \mathrm{CVC}_{\max }$ and blood triglycerides (Pearson correlation is $-0.739 ; P=0.018$ ); however, after adjusting for blood glucose, this association was no longer statistically significant (standardized $\beta$ is $-0.637 ; P=0.064$ ). There was no statistically significant association between $\% \mathrm{CVC}_{\max }$ and blood glucose in the HFM-TT trial.

\section{Discussion}

Data from the present study demonstrates that an acute high fat meal, and the resulting increase in blood triglycerides, negatively affects the plateau phase of cutaneous thermal hyperemia in young healthy participants. Our data further demonstrate that TT can mitigate the negative effects of a high fat meal. This beneficial effect lasts for at least one hour after cessation of the heat stimulus.

4.1. Initial Peak: Sensory Nerve-Mediated Cutaneous Vasodilation. The cutaneous vasodilation in response to local heating is biphasic, where cutaneous sensory nerves and TRPV-1 channels largely mediate the initial rapid vasodilation, with a modest contribution of NO ( 25\%). Data from the present study suggest that an acute high fat meal has no effect on the sensory nerve-mediated cutaneous vasodilation and that increased blood triglycerides do not affect sensory nerve 
function in young healthy humans (Figure 2). Regression analyses providing unadjusted and adjusted associations further suggests that the initial peak $\% \mathrm{CVC}_{\max }$ is not significantly correlated with either blood triglycerides or blood glucose in either of the high fat meal trials (Table 4).

As shown in Figure 2, the initial peak in the HFM-TT protocol was significantly augmented compared to both the control and high fat meal only trials. These data collectively suggest that an acute high fat meal does not negatively affect sensory nerve function and that TT can improve sensory nerve-dependent vasodilation even in young, healthy humans. Previous studies have suggested that a high fat meal and increased triglycerides can negatively affect sensory nerve function and may serve as an early risk factor for diabetic neuropathy [52-54]. It is unclear why we did not observe a detrimental effect of a high fat meal on sensory nervedependent vasodilation; however, these previous studies have been performed either in humans with type 2 diabetes or in Zucker diabetic fatty rats, which may suggest that chronic elevations in triglycerides can have detrimental effects on sensory nerve function. It is possible that the acute elevation of blood triglycerides in our study was not of sufficient duration to affect cutaneous sensory nerve function. Our data indicating that TT can augment cutaneous sensory nerve function in young, healthy humans is salient in that it suggests that this therapy may be a means to help prevent, or slow the onset of, diabetic neuropathy in individuals most at risk, such as those with prediabetes, obesity, or increased triglycerides.

\subsection{Plateau: Nitric Oxide-Mediated Cutaneous Vasodilation.} The prolonged secondary rise in CVC to a plateau during local heating is largely mediated by NO. We utilized a new local heating protocol developed by Choi et al. who showed that local heating of the skin to $39^{\circ} \mathrm{C}$, rather than the traditional $42^{\circ} \mathrm{C}$, resulted in robust, yet submaximal, cutaneous vasodilation, with a plateau phase containing a substantial $(\sim$ $80 \%)$ NO component [34]. The present study revealed that a single high fat meal significantly attenuates the plateau phase of cutaneous thermal hyperemia (Figure 3), indicating that an acute high fat meal and elevated blood triglycerides negatively affect cutaneous endothelial-dependent vasodilation.

Consumption of a high fat meal resulted in an increase in both blood triglycerides and blood glucose under both the thermoneutral and TT conditions; the attenuated plateau CVC could thus be due to either the increase in blood triglycerides or blood glucose or both. Plateau CVC in both the high fat meal and TT trials showed a strong negative correlation with blood triglycerides while there was no significant correlation with blood glucose (Table 4). Based on the standardized beta coefficients from the regression analysis, it appears that the decrement in plateau $\% \mathrm{CVC}_{\max }$ following consumption of a high fat meal is associated with the increase in blood triglycerides rather than the increase in blood glucose. It is important to note that prospective studies and data are required to determine a causative role for blood triglycerides influencing microvascular function. Nevertheless, standardized beta coefficients in this study suggest that, for every one standard deviation increase in blood triglycerides, there is a 0.879 standard deviation decrease in $\% \mathrm{CVC}_{\max }$, after controlling for blood glucose. High levels of circulating triglycerides immediately after a high fat meal have a strong, detrimental effect on cutaneous microvascular vasodilation. Inasmuch as the plateau phase is $\sim 70-80 \%$ NO-dependent, this indicates that increased triglycerides following a high fat meal negatively affect endothelial NO-dependent vasodilation. It is of interest that there was no significant correlation between plateau CVC and blood triglycerides or blood glucose during the control trial, suggesting that basal/fasting blood triglycerides and blood glucose do not affect cutaneous microvascular responses; however, there is a strong negative correlation following consumption of a high fat meal that significantly increases blood triglycerides. Although the decrement in cutaneous microvascular function following a high fat meal is associated with blood triglycerides, we cannot exclude the possibility of more complex interactions occurring at the second messenger level. For example, protein kinase $\mathrm{C}$ theta activation elicits vasoconstriction and has been shown to be associated with obesity and insulin resistance; the protein kinase $\mathrm{C}$ theta-mediated vasoconstriction occurs through a complex series of events, including Akt inhibition and ERK1/2 [55]. Whether similar complex interactions affect microvascular endothelial function in response to a high fat meal requires further investigation.

It is possible that TT improved cutaneous microvascular endothelial function following a high fat meal either by increasing bioavailable NO secondary to reduced oxidative stress or by directly increasing NO via a shear stress mechanism. High fat meals have been shown to increase oxidative stress [22, 23, 25, 56] where inflammation associated with a high fat meal causes triglyceride-rich lipoproteins to accumulate in the blood. In humans, impaired flowmediated dilation, a measure of conduit artery function and, presumably, NO-dependent vasodilation, associated with serum triglycerides has been shown to be reversed by administration of vitamins $\mathrm{C}$ and $\mathrm{E}$ [57-59]; antioxidant properties of vitamins $\mathrm{C}$ and $\mathrm{E}$ appear to counteract oxidative stress induced by a high fat meal [57-60]. Human studies have further demonstrated an increase in exhaled nitric oxide, an indicator of airway inflammation, and reduced pulmonary function following a high fat meal $[27,30]$. In the human skin microvasculature, antioxidant treatment was shown to mitigate the detrimental effects of a high fat meal [23, 25]. The augmented plateau CVC responses in the TT trial of the present study may therefore be the result of reduced oxidative stress, which restores sensory nerve function and results in reduced bioavailable NO. Studies in both humans and animals have shown that sauna therapy can improve endothelial function, increase bioavailable $\mathrm{NO}$, and reduce oxidative stress and inflammation $[43,61]$.

Exposure to TT may also improve cutaneous vascular responses following a high fat meal by directly increasing NO production. Increased skin blood flow is a direct result of increased core temperature and the resultant shear stress from increased blood flow through the cutaneous microvasculature may act as a stimulus for additional NO bioavailability [61, 62]. Indeed, sauna therapy has been shown to 
improve endothelial function in patients with risk factors for coronary heart disease and in chronic heart failure $[45,63]$. Whether shear stress is an important stimulus for $\mathrm{NO}$ in the cutaneous microvasculature remains equivocal as reactive hyperemia, a stimulus known to significantly elevate shear stress, does not appear to be an NO-dependent response in the cutaneous microvasculature of humans $[64,65]$. It is thus possible that the improvements in microvascular function observed in this study following TT are independent of a shear stress mechanism.

Regression analysis further suggests that TT improves both initial peak and plateau CVC independent of decreases in either blood triglycerides or blood glucose. In both of the high fat meal trials, blood triglycerides peaked approximately 60 minutes after the high fat meal and blood glucose peaked approximately 30 minutes after the high fat meal and there was no difference in the magnitude of blood triglycerides or blood glucose between control and TT conditions. Exposure to TT augmented both initial peak and plateau CVC independent of alterations (i.e., reductions) in either blood triglycerides or blood glucose. Based on these observations, the beneficial effects of TT appear to occur at the local neurovascular level rather than by affecting metabolism (production and/or clearance) of triglycerides and glucose. It is also possible the augmented CVC during the TT trial could have been driven by elevations in baseline following the mild heat stress protocol; however, baseline values just prior to the local heating protocol were not different than baseline in either the control or high fat meal only protocol, which would argue against an elevation in baseline for the augmented CVC responses in the TT protocol (Table 3).

4.3. Participant Demographics. Data from previous studies suggest that race/ethnicity can affect the vascular response to a high fat meal $[23,25,47,48]$. Previous studies have also shown that vascular function is greatly compromised in Asians compared to Caucasians following a high fat meal [23, 25, 47, 48]. Yet, Asians have a more pronounced response to antioxidants than Caucasians. Caucasians also appear to have a normal thermal hyperemic response following a high fat meal [47-49]. In the present study, we found that a high fat meal compromises the thermal hyperemic response in a predominantly Caucasian $(n=6,75 \%)$ participant population. The differences in findings between previous studies and the present study are most likely due to methodological differences. The previous studies heated the skin for 6 minutes, whereas our local heating protocol lasted $\sim 30$ minutes.

In this study, we chose to investigate the effect of a single high fat meal and TT on young, apparently healthy participants. To our knowledge, there are no reports on the effect of a high fat meal on both sensory nerve- and NOdependent cutaneous vasodilation simultaneously. There are also no reports regarding the combined effects of a high fat meal and TT on cutaneous neurovascular function in young, healthy participants. Pathologies such as diabetes and cardiovascular disease are known to attenuate both sensory nerve- and NO-dependent cutaneous vasodilation. To avoid potential confounding influences of pathology on cutaneous vasodilation, we chose to utilize a young, healthy population. Whether the observations from the present study translate to individuals with cardiometabolic diseases remains to be determined.

\section{Limitations}

Rapid, nonpainful local skin heating has been widely used as a noninvasive means to assess microvascular endothelial function in humans. While we based our protocol on a previously established and tested heating protocol, we did not specifically block sensory nerves or inhibit the production of $\mathrm{NO}$ in this study and therefore we cannot be certain that our results are entirely reflective of reductions in sensory nerve-dependent vasodilation or endothelial NOdependent vasodilation. In order to directly assess the effect of a high fat meal and circulating triglycerides, sensory nerve block via EMLA cream and intradermal infusion of L-NAME, a nonspecific NOS inhibitor, via microdialysis, would need to be performed. Intradermal microdialysis is minimally invasive, but it may not be a feasible means to assess microvascular endothelial function in clinical settings; a relatively simple, noninvasive test, such as nonpainful local heating, can be easily implemented in clinical settings to assess both sensory nerve and endothelial function in a wide range of populations. Despite this limitation, our data demonstrate that a single high fat meal can indeed blunt microvascular vasodilation and we are confident that using the local heating protocol developed by Choi et al. [34] represents a noninvasive means by which to assess microvascular function in humans. A second limitation to the present study is the small sample size. It is possible that some of the associations assessed by linear regression did not reach statistical significance due to the small sample size.

\section{Conclusion}

We found that a single high fat meal attenuated both initial peak and plateau CVC responses to local heating compared to control while exposure to TT prior to, during, and after the consumption of a high fat meal restored cutaneous vascular responses. A novel aspect of this study is the use of local heating to simultaneously assess sensory nerve function and endothelial function in response to a high fat meal. These data indicate that an acute high fat meal negatively affects microvascular endothelial function while the application of TT can restore microvascular endothelial function.

\section{Competing Interests}

There are no competing interests regarding the publication of this paper. 


\section{Authors' Contributions}

This study was performed by Jennifer Harvey in partial fulfillment of the degree of Master of Science in the Department of Kinesiology and Health at Georgia State University. Jennifer Harvey was responsible for experimental design, data collection, data analysis and interpretation, and drafting the paper. Bruno Roseguini was responsible for experimental design, data collection, data analysis and interpretation, and drafting the paper. Benjamin M. Goerger was responsible for data analysis and interpretation and editing the paper. Elizabeth Fallon was responsible for statistical analysis, data analysis and interpretation, and editing the paper. Brett Wong was responsible for experimental design, data collection, data analysis and interpretation, and drafting the paper.

\section{Acknowledgments}

The authors acknowledge the participants for their time and dedication in completing the protocol. The authors thank Ms. Taylor Copeland and Mr. Namaan Smith for their assistance with data collection.

\section{References}

[1] J.-H. Bae, E. Bassenge, K.-B. Kim et al., "Postprandial hypertriglyceridemia impairs endothelial function by enhanced oxidant stress," Atherosclerosis, vol. 155, no. 2, pp. 517-523, 2001.

[2] J.-H. Bae, E. Bassengel, H.-J. Lee et al., "Impact of postprandial hypertriglyceridemia on vascular responses in patients with coronary artery disease: effects of ACE inhibitors and fibrates," Atherosclerosis, vol. 158, no. 1, pp. 165-171, 2001.

[3] J.-H. Bae, M. Schwemmer, I.-K. Lee et al., "Postprandial hypertriglyceridemia-induced endothelial dysfunction in healthy subjects is independent of lipid oxidation," International Journal of Cardiology, vol. 87, no. 2-3, pp. 259-267, 2003.

[4] M. P. De Boer, R. I. Meijer, N. J. Wijnstok et al., "Microvascular dysfunction: a potential mechanism in the pathogenesis of obesity-associated insulin resistance and hypertension," Microcirculation, vol. 19, no. 1, pp. 5-18, 2012.

[5] M. P. de Boer, N. J. Wijnstok, E. H. Serné et al., "Body mass index is related to microvascular vasomotion, this is partly explained by adiponectin," European Journal of Clinical Investigation, vol. 44, no. 7, pp. 660-667, 2014.

[6] A. Lerman and A. M. Zeiher, "Endothelial function: cardiac events," Circulation, vol. 111, no. 3, pp. 363-368, 2005.

[7] E. H. Serné, C. D. A. Stehouwer, J. C. Ter Maaten et al., "Microvascular function relates to insulin sensitivity and blood pressure in normal subjects," Circulation, vol. 99, no. 7, pp. 896902, 1999.

[8] T. F. T. Antonios, D. R. J. Singer, N. D. Markandu, P. S. Mortimer, and G. A. MacGregor, "Rarefaction of skin capillaries in borderline essential hypertension suggests an early structural abnormality," Hypertension, vol. 34, no. 4, pp. 655-658, 1999.

[9] J. Borén, N. Matikainen, M. Adiels, and M.-R. Taskinen, "Postprandial hypertriglyceridemia as a coronary risk factor," Clinica Chimica Acta, vol. 431, pp. 131-142, 2014.

[10] B. Gabriel, A. Ratkevicius, P. Gray, M. P. Frenneaux, and S. R. Gray, "High-intensity exercise attenuates postprandial lipaemia and markers of oxidative stress," Clinical Science, vol. 123, no. 5, pp. 313-321, 2012.
[11] L. A. Holowatz, C. S. Thompson-Torgerson, and W. L. Kenney, "The human cutaneous circulation as a model of generalized microvascular function," Journal of Applied Physiology, vol. 105, no. 1, pp. 370-372, 2008.

[12] J. P. Noon, B. R. Walker, D. J. Webb et al., "Impaired microvascular dilatation and capillary rarefaction in young adults with a predisposition to high blood pressure," The Journal of Clinical Investigation, vol. 99, no. 8, pp. 1873-1879, 1997.

[13] E. H. Serné, R. O. B. Gans, J. C. Ter Maaten, P. M. ter Wee, A. J. M. Donker, and C. D. A. Stehouwer, "Capillary recruitment is impaired in essential hypertension and relates to insulin's metabolic and vascular actions," Cardiovascular Research, vol. 49, no. 1, pp. 161-168, 2001.

[14] M. Herieka and C. Erridge, "High-fat meal induced postprandial inflammation," Molecular Nutrition and Food Research, vol. 58, no. 1, pp. 136-146, 2014.

[15] A. Khor, R. Grant, C. Tung et al., "Postprandial oxidative stress is increased after a phytonutrient-poor food but not after a kilojoule-matched phytonutrient-rich food," Nutrition Research, vol. 34, no. 5, pp. 391-400, 2014.

[16] J. P. Wallace, B. Johnson, J. Padilla, and K. Mather, "Postprandial lipaemia, oxidative stress and endothelial function: a review," International Journal of Clinical Practice, vol. 64, no. 3, pp. 389403, 2010.

[17] P. Yi, J. Pang, J. S. Alexander, and C. Rivera, “The endotoxin/tolllike receptor- 4 axis mediates gut microvascular dysfunction associated with post-prandial lipidemia," BMC Physiology, vol. 13, no. 1, article 12, 2013.

[18] D. N. Atochin and P. L. Huang, "Endothelial nitric oxide synthase transgenic models of endothelial dysfunction," Pflugers Archiv European Journal of Physiology, vol. 460, no. 6, pp. 965974, 2010.

[19] M. El Assar, J. Angulo, and L. Rodríguez-Mañas, "Oxidative stress and vascular inflammation in aging," Free Radical Biology and Medicine, vol. 65, pp. 380-401, 2013.

[20] D. A. Hobbs, T. W. George, and J. A. Lovegrove, "The effects of dietary nitrate on blood pressure and endothelial function: a review of human intervention studies," Nutrition Research Reviews, vol. 26, no. 2, pp. 210-222, 2013.

[21] L. Jungersten, A. Ambring, B. Wall, and Å. Wennmalm, "Both physical fitness and acute exercise regulate nitric oxide formation in healthy humans," Journal of Applied Physiology, vol. 82, no. 3, pp. 760-764, 1997.

[22] J. Brandauer, R. Q. Landers-Ramos, N. T. Jenkins, E. E. Spangenburg, J. M. Hagberg, and S. J. Prior, "Effects of prior acute exercise on circulating cytokine concentration responses to a high-fat meal," Physiological Reports, vol. 1, no. 3, Article ID e00040, 2013.

[23] J. Petrofsky, F. Alshammari, I. A. Khowailed et al., "The effect of acute administration of vitamin D on micro vascular endothelial function in Caucasians and South Asian Indians," Medical Science Monitor, vol. 19, no. 1, pp. 641-647, 2013.

[24] J. S. Petrofsky, M. Laymon, H. Lee et al., "CoQ10 and endothelial function in Asians from Korea compared to Asians born in the United States and US born Caucasians," Medical Science Monitor, vol. 19, no. 1, pp. 339-346, 2013.

[25] J. Yim, J. Petrofsky, L. Berk et al., "Protective effect of antioxidants on endothelial function in young Korean-Asians compared to Caucasians," Medical Science Monitor, vol. 18, no. 8, pp. CR467-CR479, 2012. 
[26] S. Lupachyk, P. Watcho, N. Hasanova, U. Julius, and I. G. Obrosova, "Triglyceride, nonesterified fatty acids, and prediabetic neuropathy: role for oxidative-nitrosative stress," Free Radical Biology \& Medicine, vol. 52, no. 8, pp. 1255-1263, 2012.

[27] C. J. Ade, S. K. Rosenkranz, and C. A. Harms, "The effects of short-term fish oil supplementation on pulmonary function and airway inflammation following a high-fat meal," European Journal of Applied Physiology, vol. 114, no. 4, pp. 675-682, 2014.

[28] B. D. Johnson, J. Padilla, R. A. Harris, and J. P. Wallace, "Vascular consequences of a high-fat meal in physically active and inactive adults," Applied Physiology, Nutrition and Metabolism, vol. 36, no. 3, pp. 368-375, 2011.

[29] J. Padilla, R. A. Harris, A. D. Fly, L. D. Rink, and J. P. Wallace, "The effect of acute exercise on endothelial function following a high-fat meal," European Journal of Applied Physiology, vol. 98, no. 3, pp. 256-262, 2006.

[30] S. K. Rosenkranz, D. K. Townsend, S. E. Steffens, and C. A. Harms, "Effects of a high-fat meal on pulmonary function in healthy subjects," European Journal of Applied Physiology, vol. 109, no. 3, pp. 499-506, 2010.

[31] R. S. Bruning, L. Santhanam, A. E. Stanhewicz et al., "Endothelial nitric oxide synthase mediates cutaneous vasodilation during local heating and is attenuated in middle-aged human skin," Journal of Applied Physiology, vol. 112, no. 12, pp. 20192026, 2012.

[32] V. E. Brunt and C. T. Minson, "KCa channels and epoxyeicosatrienoic acids: major contributors to thermal hyperaemia in human skin," Journal of Physiology, vol. 590, no. 15, pp. 35233534, 2012.

[33] S. J. Carter and G. J. Hodges, "Sensory and sympathetic nerve contributions to the cutaneous vasodilator response from a noxious heat stimulus," Experimental Physiology, vol. 96, no. 11, pp. 1208-1217, 2011.

[34] P. J. Choi, V. E. Brunt, N. Fujii, and C. T. Minson, "New approach to measure cutaneous microvascular function: an improved test of NO-mediated vasodilation by thermal hyperemia," Journal of Applied Physiology, vol. 117, no. 3, pp. 277-283, 2014.

[35] G. J. Hodges and P. A. Sparks, "Contributions of endothelial nitric oxide synthase, noradrenaline, and neuropeptide $\mathrm{Y}$ to local warming-induced cutaneous vasodilatation in men," Microvascular Research, vol. 90, pp. 128-134, 2013.

[36] D. L. Kellogg Jr., Y. Liu, I. F. Kosiba, and D. O’Donnell, “Role of nitric oxide in the vascular effects of local warming of the skin in humans," Journal of Applied Physiology, vol. 86, no. 4, pp. 1185-1190, 1999.

[37] D. L. Kellogg Jr., J. L. Zhao, and Y. Wu, "Roles of nitric oxide synthase isoforms in cutaneous vasodilation induced by local warming of the skin and whole body heat stress in humans," Journal of Applied Physiology, vol. 107, no. 5, pp. 1438-1444, 2009.

[38] D. L. Kellogg Jr., J. L. Zhao, and Y. Wu, "Endothelial nitric oxide synthase control mechanisms in the cutaneous vasculature of humans in vivo," American Journal of Physiology-Heart and Circulatory Physiology, vol. 295, no. 1, pp. H123-H129, 2008.

[39] C. T. Minson, L. T. Berry, and M. J. Joyner, "Nitric oxide and neurally mediated regulation of skin blood flow during local heating," Journal of Applied Physiology, vol. 91, no. 4, pp. 16191626, 2001.

[40] B. J. Wong and S. M. Fieger, "Transient receptor potential vanilloid type-1 (TRPV-1) channels contribute to cutaneous thermal hyperaemia in humans," Journal of Physiology, vol. 588, no. 21 , pp. 4317-4326, 2010.
[41] B. J. Wong and C. T. Minson, "Altered thermal hyperaemia in human skin by prior desensitization of neurokinin-1 receptors," Experimental Physiology, vol. 96, no. 6, pp. 599-609, 2011.

[42] S. Biro, A. Masuda, T. Kihara, and C. Tei, "Clinical implications of thermal therapy in lifestyle-related diseases," Experimental Biology and Medicine, vol. 228, no. 10, pp. 1245-1249, 2003.

[43] M. Gayda, F. Paillard, P. Sosner et al., "Effects of sauna alone and postexercise sauna baths on blood pressure and hemodynamic variables in patients with untreated hypertension," Journal of Clinical Hypertension, vol. 14, no. 8, pp. 553-560, 2012.

[44] Y. Ikeda, S. Biro, Y. Kamogawa et al., "Repeated sauna therapy increases arterial endothelial nitric oxide synthase expression and nitric oxide production in cardiomyopathic hamsters," Circulation Journal, vol. 69, no. 6, pp. 722-729, 2005.

[45] T. Kihara, S. Biro, M. Imamura et al., "Repeated sauna treatment improves vascular endothelial and cardiac function in patients with chronic heart failure," Journal of the American College of Cardiology, vol. 39, no. 5, pp. 754-759, 2002.

[46] M. Imamura, S. Biro, T. Kihara et al., "Repeated thermal therapy improves impaired vascular endothelial function in patients with coronary risk factors," Journal of the American College of Cardiology, vol. 38, no. 4, pp. 1083-1088, 2001.

[47] C. Bui, J. Petrofsky, L. Berk, D. Shavlik, W. Remigio, and S. Montgomery, "Acute effect of a single high-fat meal on forearm blood flow, blood pressure and heart rate in healthy male Asians and Caucasians: a pilot study," Southeast Asian Journal of Tropical Medicine and Public Health, vol. 41, no. 2, pp. 490500, 2010.

[48] J. S. Petrofsky, F. Alshammari, G. S. Bains et al., "What is more damaging to vascular endothelial function: diabetes, age, high BMI, or all of the above?" Medical Science Monitor, vol. 19, no. 1, pp. 257-263, 2013.

[49] J. Yim, J. Petrofsky, L. Berk, N. Daher, and E. Lohman, "Differences in endothelial function between Korean-Asians and Caucasians," Medical Science Monitor, vol. 18, no. 6, pp. CR337-CR343, 2012.

[50] L. A. Holowatz, C. S. Thompson, C. T. Minson, and W. L. Kenney, "Mechanisms of acetylcholine-mediated vasodilation in young and aged human skin," Journal of Physiology, vol. 563, no. 3, pp. 965-973, 2005.

[51] C. T. Minson, "Thermal provocation to evaluate microvascular reactivity in human skin," Journal of Applied Physiology, vol. 109, no. 4, pp. 1239-1246, 2010.

[52] S. Lupachyk, P. Watcho, N. Hasanova, U. Julius, and I. G. Obrosova, "Triglyceride, nonesterified fatty acids, and prediabetic neuropathy: role for oxidative-nitrosative stress," Free Radical Biology and Medicine, vol. 52, no. 8, pp. 1255-1263, 2012.

[53] H. Shevalye, S. Lupachyk, P. Watcho et al., "Prediabetic nephropathy as an early consequence of the high-calorie/highfat diet: relation to oxidative stress," Endocrinology, vol. 153, no. 3, pp. 1152-1161, 2012.

[54] A. G. Smith and J. R. Singleton, "Obesity and hyperlipidemia are risk factors for early diabetic neuropathy," Journal of Diabetes and Its Complications, vol. 27, no. 5, pp. 436-442, 2013.

[55] W. Bakker, P. Sipkema, C. D. A. Stehouwer et al., "Protein kinase C $\theta$ activation induces insulin-mediated constriction of muscle resistance arteries," Diabetes, vol. 57, no. 3, pp. 706-713, 2008.

[56] P. C. Calder, N. Ahluwalia, F. Brouns et al., "Dietary factors and low-grade inflammation in relation to overweight and obesity," British Journal of Nutrition, vol. 106, no. 3, pp. S5-S78, 2011. 
[57] K. Esposito, F. Nappo, F. Giugliano, G. Giugliano, R. Marfella, and D. Giugliano, "Effect of dietary antioxidants on postprandial endothelial dysfunction induced by a high-fat meal in healthy subjects," The American Journal of Clinical Nutrition, vol. 77, no. 1, pp. 139-143, 2003.

[58] G. D. Plotnick, M. C. Corretti, and R. A. Vogel, "Effect of antioxidant vitamins on the transient impairment of endotheliumdependent brachial artery vasoactivity following a single highfat meal," Journal of the American Medical Association, vol. 278, no. 20, pp. 1682-1686, 1997.

[59] R. A. Vogel, M. C. Corretti, and G. D. Plotnick, “The postprandial effect of components of the mediterranean diet on endothelial function," Journal of the American College of Cardiology, vol. 36, no. 5, pp. 1455-1460, 2000.

[60] G. C. Burdge and P. C. Calder, "Plasma cytokine response during the postprandial period: a potential causal process in vascular disease?" British Journal of Nutrition, vol. 93, no. 1, pp. 3-9, 2005.

[61] S. Fujita, Y. Ikeda, M. Miyata et al., "Effect of Waon therapy on oxidative stress in chronic heart failure," Circulation Journal, vol. 75, no. 2, pp. 348-356, 2011.

[62] M. L. Pall, "Do sauna therapy and exercise act by raising the availability of tetrahydrobiopterin?" Medical Hypotheses, vol. 73, no. 4, pp. 610-613, 2009.

[63] M. Imamura, S. Biro, T. Kihara et al., "Repeated thermal therapy improves impaired vascular endothelial function in patients with coronary risk factors," Journal of the American College of Cardiology, vol. 38, no. 4, pp. 1083-1088, 2001.

[64] B. J. Wong, B. W. Wilkins, L. A. Holowatz, and C. T. Minson, "Nitric oxide synthase inhibition does not alter the reactive hyperemic response in the cutaneous circulation," Journal of Applied Physiology, vol. 95, no. 2, pp. 504-510, 2003.

[65] J. L. Zhao, P. E. Pergola, L. J. Roman, and D. L. Kellogg Jr., "Bioactive nitric oxide concentration does not increase during reactive hyperemia in human skin," Journal of Applied Physiology, vol. 96, no. 2, pp. 628-632, 2004. 


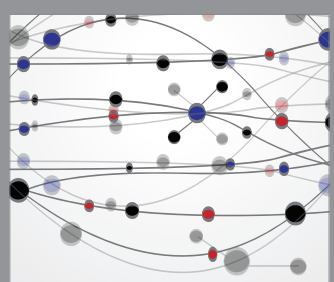

The Scientific World Journal
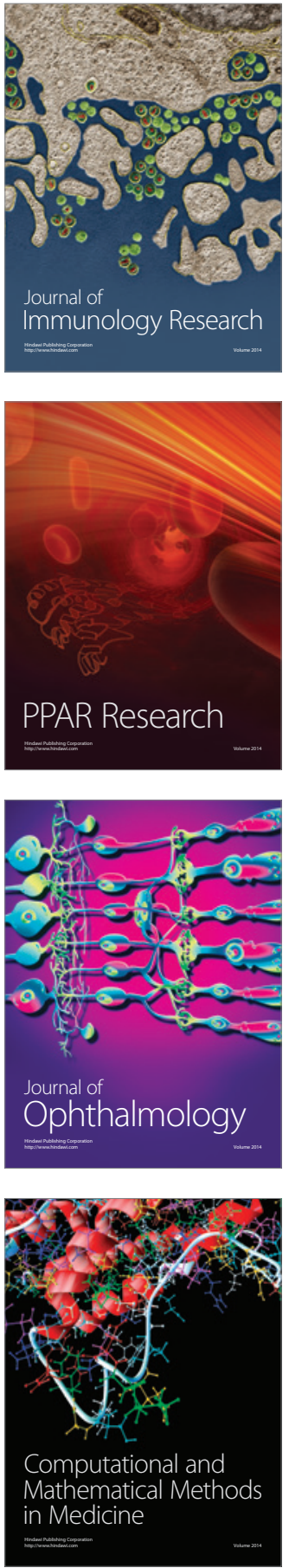

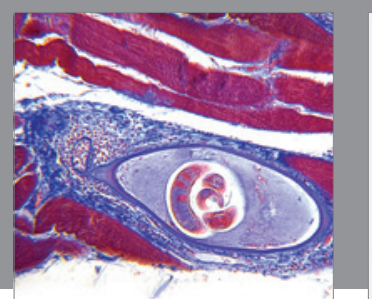

Gastroenterology Research and Practice

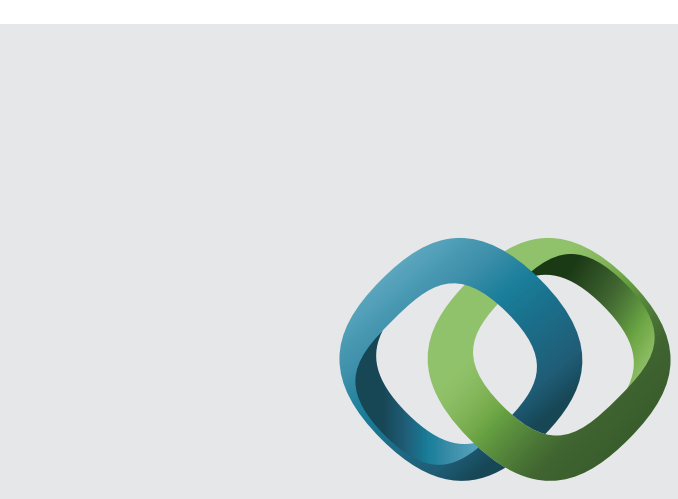

\section{Hindawi}

Submit your manuscripts at

http://www.hindawi.com
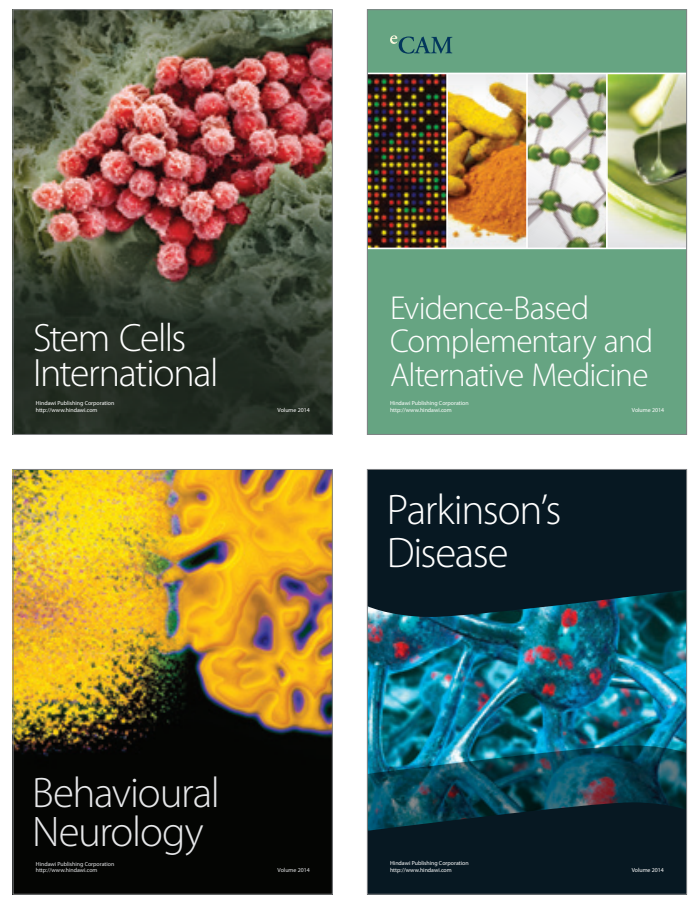
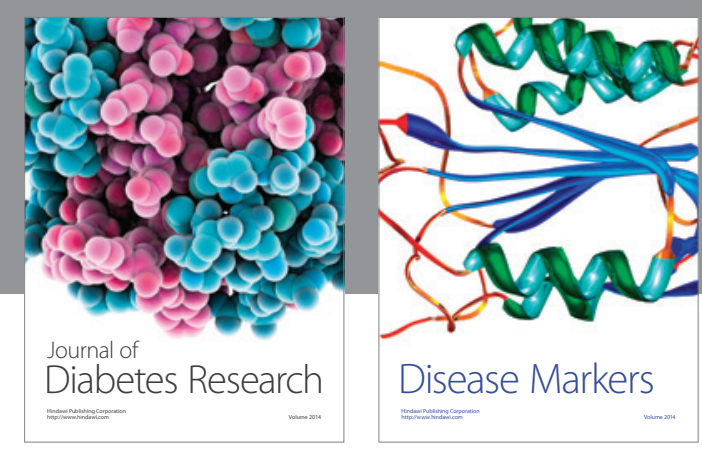

Disease Markers
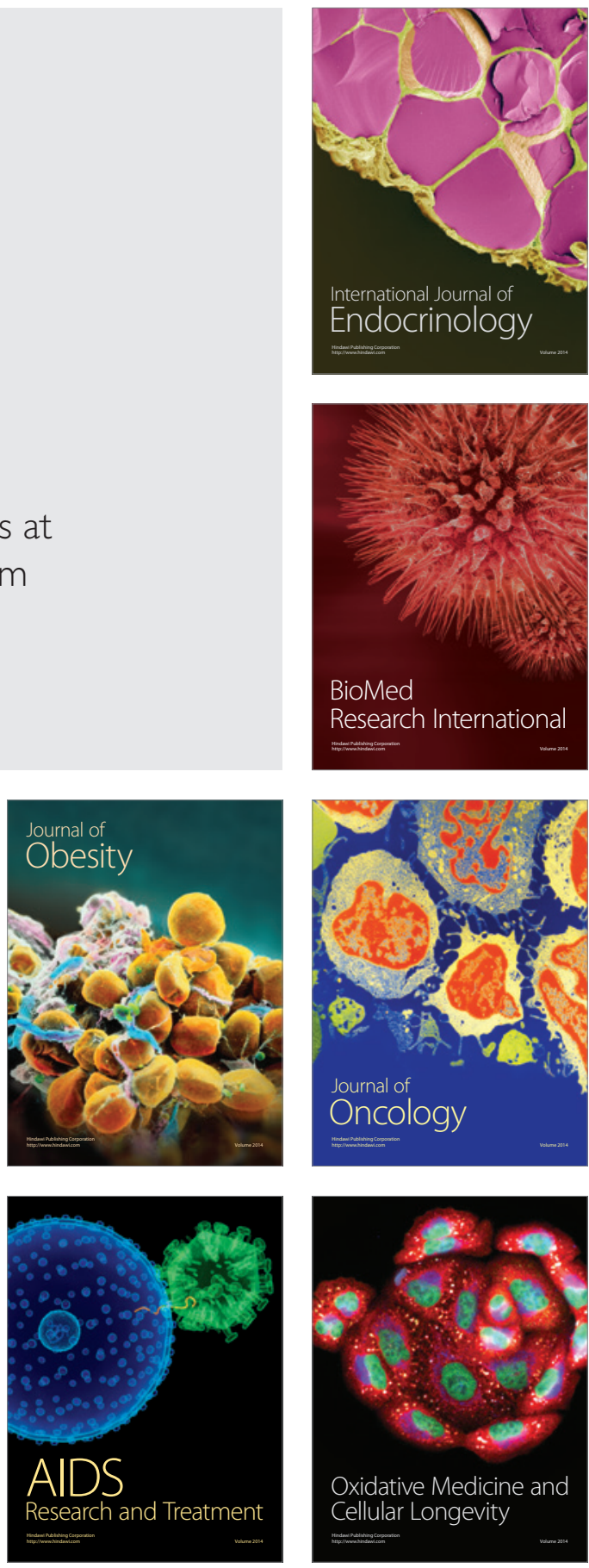